\title{
INCIDENCIA DE LA COMPRA DE VOTO \\ EN LAS ELECCIONES MEXICANAS DE 2018: \\ DISCUSIÓN SOBRE MEDICIÓN, MÉTODO Y RETOS \\ DE LOS ESTUDIOS ELECTORALES
}

Pablo Parás García ${ }^{1}$

En México se han realizado paneles electorales en cada elección presidencial desde el año $2000{ }^{2}$ En la elección de 2018 la investigación tuvo dos olas: la primera preelectoral en junio y la segunda en agosto/septiembre después de las elecciones de julio. El trabajo de campo estuvo a cargo de Data OPM y el tema principal de investigación fue la compra de voto. ${ }^{3}$ La pregunta principal que guía la presente discusión es la siguiente: ies importante incluir la medición de la incidencia de la compra de voto (en países latinoamericanos) como un componente relevante de los estudios de comportamiento electoral? Para intentar una respuesta se pretende discutir tres temas específicos: (a) la medición de la incidencia de la compra de voto; (b) el impacto de la inseguridad en el trabajo de campo y los estudios tipo panel; y (c) las carencias/retos de los estudios electorales.

Sobre la medición de la incidencia hay dos aspectos importantes que cubrir. Uno tiene que ver con la dificultad de medir un tema potencialmente "sensible" y otro con la profundidad y la suficiencia de la medición del fenómeno.

\footnotetext{
${ }^{1}$ OPM y Georgetown University.

2 Todos son públicos. Datos e información sobre los paneles de 2000, 2006 y 2012 pueden ser consultados en: https://mexicopanelstudy.mit.edu.

${ }^{3}$ Los investigadores principales del panel electoral 2018 son Kenneth Green y Alberto Simpser con la colaboración de Carlos López y el autor. La primera ola del estudio fue patrocinada por Acción Ciudadana frente a la pobreza y la segunda por el Instituto Federal Electoral.
} 
La medición de fenómenos que implican que el individuo acepte o reporte comportamientos que pueden ser considerados socialmente indeseables o hasta conductas ilegales presenta claros desafíos para el diseño de encuesta. Como ejemplos tenemos la medición del consumo de drogas, la corrupción y la compra de voto, fenómenos que pueden ser subestimados si no se cuenta con un diseño que mitigue dicha sensibilidad al momento de ser reportados por los entrevistados. En el caso de la compra del voto puede existir además un elemento de coacción con el cual se condicione la participación de la persona en programas sociales u otros beneficios actuales o futuros. Hasta ahora se han usado algunas técnicas para enfrentar estas dificultades, como los experimentos de lista o la redacción de la pregunta por la cual el individuo es más "víctima" que "participante". ${ }^{4}$ Más adelante se presentan cifras de incidencia comparativa que sugieren que dichas técnicas parecieran no ser suficientes para eliminar el problema de subestimación del fenómeno. Adicionalmente es muy común que la autoridad electoral realice importantes campañas contra la compra de voto en medios masivos de comunicación.

En cuanto a la suficiencia y profundidad de la medición de la incidencia de compra de voto podemos afirmar que hay un problema de cobertura. Me explico. La compra de voto, una modalidad del clientelismo, es en sí un concepto potencialmente complejo cuya medición en suficiencia y profundidad requiere el uso de múltiples reactivos. ${ }^{5}$ Específicamente sobre la incidencia y magnitud de la compra de voto, es necesario conocer quién y qué ha recibido, pero también si hubo "ofertas" rechazadas o promesas aún no cumplidas, e idealmente preguntar esto sobre todos los miembros (adultos) del hogar. Este modelo RRP (Recibe + Rechaza + Promesa aún no dan) constituye la ba-

\footnotetext{
4 Para una discusión a detalle ver: https://integralia.com.mx/fortalezasy debilidades/Capitulo 15-Simpser.pdf.

${ }^{5}$ Idem.
} 
se del instrumento de medición del panel electoral México 2018. A cada persona entrevistada se le preguntó primero sobre su experiencia directa y luego sobre la experiencia de otros miembros del hogar, de tal suerte que cada individuo (y hogar) pudo ser clasificado en una de cuatro categorias: como alguien que recibió; le ofrecieron y lo rechazó; le prometieron y aún no le dan; o ninguna de la anteriores. La suma de las tres primeras categorias constituye una medición suficiente de la incidencia de la compra de voto en México. Adicionalmente es relevante conocer quién solicita, quién ofrece, a cambio de qué se ofrece, si se corren riesgos de incumplimiento, el valor relativo de lo dado/ofrecido, el conocimiento o cercanía con la persona que lo da/ofrece, qué tan común es el fenómeno en su colonia, la incidencia en elecciones pasadas y otros indicadores del fenómeno que permitan conocerlo mejor y evaluar su posible impacto sobre el comportamiento electoral. Todos los indicadores hasta aquí mencionados fueron incluidos también en la medición, con lo cual es probable que el panel electoral México 2018 sea una de las mediciones más completas y profundas de la compra de voto en Latinoamérica.

En el panel electoral México 2018, la principal estrategia para mitigar la sensibilidad y tener una medición más precisa de la incidencia de compra de voto consistió en que el entrevistado respondiera las preguntas en forma confidencial con formato autoaplicado. La entrevista fue personal en el domicilio del entrevistado en modo CAPI (Computer Aided Personal Interview, por sus siglas en inglés) usando dispositivos electrónicos tipo tableta. La selección, introducción e inicio del instrumento fueron aplicados de forma tradicional: el entrevistador lee las preguntas al entrevistado y espera y registra las respuestas. Al llegar a la sección del cuestionario con las preguntas sobre compra de voto ly en una instancia previa para que el entrevistado se familiarizara con el uso de la tableta) se leía la siguiente instrucción/introducción: 
[Leer lento y claro] Ahora quisiera preguntarle sobre sus propias experiencias durante el 2018. A veces, los partidos políticos regalan despensas, dinero en efectivo, tarjetas, materiales para construcción, tinacos, medicinas; o bien facilitan el acceso a programas de gobierno como Prospera o Seguro Popular, dan becas escolares, o proveen atención médica. También pueden ofrecer trabajos o facilitar trámites. En las siguientes preguntas, quisiera conocer su experiencia al respecto durante el 2018. Para garantizar su privacidad, le voy a entregar mi dispositivo para que conteste en privado algunas preguntas. Como antes, una vez que seleccione cada respuesta, oprima el cuadro verde. Cuando termine, entrégueme el dispositivo. Le recuerdo que todas sus respuestas son confidenciales.

[Encuestador: Entregar el dispositivo, mostrar el cuadro verde, voltearse y recoger el dispositivo cuando el encuestado haya terminado.]

Esta redacción tenía cuatro propósitos: introducir las principales/múltiples prácticas que constituyen la compra de voto, enmarcar el fenómeno como una acción protagonizada o iniciada por los partidos políticos (no el individuo), recordar al entrevistado el compromiso de confidencialidad del uso de los datos e instruir el uso del dispositivo en forma privada (i.e. la técnica autoaplicada).

¿Cuál es la incidencia de la compra del voto en México medida por el panel electoral México 2018 usando la técnica autoaplicada y el modelo RRP, y de que tamaño es la subestimación en estudios anteriores? El panel electoral México 2012 contiene un reactivo que mide de forma general el fenómeno con la siguiente redacción: "En las últimas semanas, ¿Alguien le hizo un favor o le ofreció un regalo o 
servicio a cambio de su voto?". ${ }^{6}$ Aunque esta pregunta no parece intrusiva, agresiva o potencialmente sensible, registra incidencia de compra de voto que se antoja baja: $2,7 \%$ de los entrevistados respondieron afirmativamente en la ola preelectoral y 5,7\% en la ola poselectoral. Un dato más reciente es el de ola 2014 del Barómetro de las Américas - Lapop que contiene el siguiente reactivo: "Y pensando en las últimas elecciones presidenciales de 2012, ¿alguien le ofreció a usted un favor, regalo o beneficio a cambio de su voto?". Este reactivo aplicado en otro momento en el tiempo (más alejado de la elección) pero con una pregunta muy parecida arroja una incidencia significativamente diferente: 14,5\% afirmó haber recibido una oferta clientelar y, en una pregunta más general del mismo estudio, donde se pregunta sobre si conoce a alguien a quien se haya hecho la oferta, el porcentaje se incrementa al $23 \%{ }^{7}$

\section{Incidencia de la compra de voto México 2018}

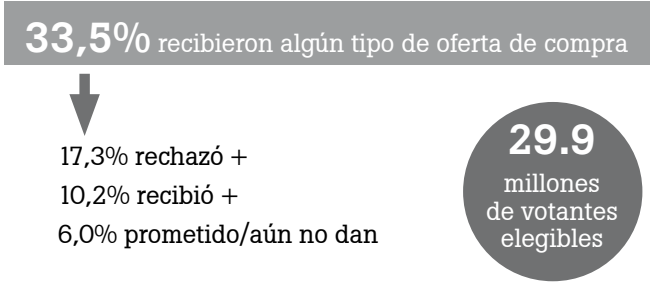

Usando la técnica autoaplicada y el modelo RRP los datos de la elección del 2018 arrojan datos muy diferentes. Al preguntar sobre la experiencia directa del entrevistado en la ola prelectoral, obtenemos que en total un tercio

\footnotetext{
${ }^{6}$ Adicionalmente, el panel electoral México 2012 pregunta qué le ofrecieron y quién lo ofreció. Los datos se pueden consultar en: http://mexicopanelstudy.mit.edu/.

${ }^{7}$ Se preguntó: "Pensando en las últimas elecciones nacionales, ¿algún candidato $o$ alguien de un partido político le ofreció un favor, regalo u otro beneficio a alguna persona que usted conoce para que lo apoye o vote por él?". Los datos se pueden consultar en: https://www.vanderbilt.edu/lapop/.
} 
de los entrevistados (33,5\%) afirma haber recibido ofertas con una composición muy interesante: 17,3\% recibió una oferta que rechazó, 10,2\% recibió y aceptó la oferta y a un $6,0 \%$ se le hizo una promesa que aún no se materializó. La incidencia medida con esta técnica/modelo es significativamente superior a las incidencias del panel 2012 y la ola 2014 de Lapop, lo cual sugiere que el fenómeno había sido subestimado en el pasado. De acuerdo con estos datos la proyección es que en el 2018 el fenómeno afectó a 29,9 millones de electores.

Como se mencionó arriba, el estudio contiene indicadores adicionales que en su conjunto constituyen una medición a profundidad del fenómeno. ${ }^{8}$ A continuación una breve discusión de los principales. Sorprende que aun cuando

$79 \%$ dice

que no hay

problema en

votar por un

partido

diferente al

oferente

la incidencia de la compra de voto es considerable, ocho de cada diez de los involucrados (79\%) dice que no hay problema por votar por un partido distinto al oferente; es decir, pareceria que en la mayoria de los casos el partido que ofrece no tiene forma de hacer cumplir u obligar al ciudadano. De entre quienes mencionan que sí pueden tener problemas por incumplimiento, los principales mencionados son la pérdida de empleo, el no recibir los regalos/favores/servicios, agresión física, enojo de amigos o familiares y sentirse mal por no cumplir. De acuerdo con los entrevistados lo que se da/ofrece son principalmente despensas, dinero, materiales de construcción y gorras/playeras. En términos comparativos, la incidencia de ofertas de becas o pertenencia a programas sociales (o amenaza de eliminación de ellos) es mucho menor. Lo que más se solicita a cambio de lo ofrecido (como era de suponerse) es votar por el partido seguido de información sobre la credencial de elector, o quedarse con esta. ¿Qué partidos están involucrados en la compra

${ }^{8}$ Los resultados de la ola prelectoral pueden ser consultado en: http:// democraciasinpobreza.mx/Informe.pdf. 
de voto? La respuesta es que todos, pero en diferente magnitud. Todos los partidos (en forma individual o como coalición) resultaron señalados en la actividad clientelar, pero se registró una mayor actividad de los tradicionales/prin-

$31 \%$

conocia bien/

muy bien a

quien hizo

la oferta cipales (i.e. PRI, PAN y PRD) en comparación al más reciente y ganador de la elección, Morena. Lo más interesante es que la principal respuesta fue que "múltiples" partidos estuvieron involucrados, es decir, que más de uno llega o compite por el elector y este considera simultáneamente ofertas de varios actores. Por último, compartimos datos sobre el valor de la transacción y la cercanía con el actor. Sobre el primero podemos decir que el 11,8\% de quienes rechazaron/recibieron/les prometieron, consideran que les es difícil o imposible acceder a lo prometido; sobre la cercania, un tercio (31\%) de este mismo grupo señala que conocía muy bien o bien a la persona que hizo la oferta.

Es importante señalarle al lector la efectividad de la técnica autoaplicada. Como es de suponerse, no todos los entrevistados aceptan, pueden usar la tableta o sentirse cómodos usándola en forma individual para responder las preguntas. Seis de cada diez $(57,9 \%)$ respondieron todas las preguntas en forma privada en modo autoaplicado; el $17,6 \%$ lo hizo en forma parcial y un cuarto $(24,5 \%)$ no aceptó responder las preguntas y solicitó que se le aplicaran las preguntas en forma convencional. Aún no contamos con el análisis de diferencias de la incidencia entre estos subgrupos para evaluar el impacto de la técnica/modelo de aplicación, lo cual resultará de suma relevancia para futuras investigaciones.

Hasta aquí la discusión sobre la medición de la incidencia de la compra de voto desde la experiencia del panel electoral México 2018. Pasamos ahora a la discusión del impacto de la inseguridad en el trabajo de campo y los estudios tipo panel y las carencias/retos de los estudios electorales. La inseguridad en México se ha incrementado cons- 
tante y significativamente en los últimos quince años y esto ha tenido un impacto directo en los estudios demoscópicos. En 2009 recibí una llamada de una reportera de un periódico nacional indagando sobre las áreas del país que yo consideraba no serian cubiertas en el censo de 2010. La pregunta sugeria que una operación de campo tan potente y con tantos recursos como la de la oficina del censo de México no tenía capacidad o voluntad de cubrir en su totalidad el territorio mexicano; sugería que el gobierno quizá no era capaz de cumplir una tarea tan básica como contar a todos sus habitantes. En términos comparativos, la operación de campo de la empresa que dirijo es mucho menos potente y mucho más vulnerable. La inseguridad tiene varios efectos perversos directos en las encuestas de opinión pública. El más importante es, por supuesto, el riesgo del capital humano, pero desde el punto de vista del método y la precisión, la seguridad puede estar teniendo un importante impacto ligado a la representatividad y selección de la muestra. A continuación ofrezco algunos ejemplos para ilustrar la magnitud del impacto de la inseguridad.

Data OPM es una empresa pequeña que realiza alrededor de treinta y cinco proyectos de investigación al año. Durante 2016 y 2017 sustituimos por razones de seguridad, en promedio, el 4,5\% de las unidades primarias muestrales (UPM) de estos estudios, ya sea al inicio de la selección de la muestra con conocimiento de que la UPM representaba una zona riesgosa o una vez que la brigada de trabajo de campo llegaba a la UPM y constataba el riesgo del lugar. Durante 2018 el promedio se ha triplicado y se encuentra por encima del 15\%, siendo mayor en los estudios más recientes. Adicionalmente, el tipo de riesgo que corren los equipos ha pasado de robos de equipo y amenazas menores a incidentes de gran seriedad e impacto como el secuestro y riesgo de linchamientos. Por primera vez en quince años de operación en 2018 sufrimos tres eventos de gran riesgo que pusieron en peligro la integridad física de nuestros en- 
trevistadores. En la ola poselectoral del panel electoral de 2012 se logró reentrevistar al 70\% de los entrevistados; en 2018, con procesos e información más sofisticada, solo al $45 \% .{ }^{9}$ Parte de la explicación de esta reducción tiene que ver con la inseguridad que genera desconfianza y menor disposición de los individuos a participar y compartir información con los entrevistadores. Considero de suma importancia que desde Wapor Latinoamérica se tenga una discusión seria y profunda sobre el impacto que está teniendo la inseguridad en el proceso de investigación de la opinión pública en los paises de la región.

Un reto adicional de los estudios electorales, agravante de la situación de inseguridad hasta aquí descrita, es lo que llamo el "problema de la prisa". A diferencia de otras elecciones presidenciales en México, la de 2018 no fue un fenómeno difícil de medir. La ventaja entre el candidato puntero y los demás estuvo marcada desde un principio y fue creciendo. La mayoria del electorado no tuvo problema en declarar sus preferencias, las que fueron captadas con aceptable precisión por las casas encuestadoras serias de nuestro país. Sin embargo, la mayoría de las encuestas electorales, patrocinadas casi en su totalidad por partidos políticos o medios de comunicación, siguen una lógica electoral en la que se busca tener mediciones frecuentes durante la contienda.

Desde el punto de vista de la robustez científica, esto genera un incentivo perverso: los usuarios de encuestas electorales necesitan contar con datos frecuentes y generados en períodos de levantamiento cortos. Un dato al inicio de campaña, otro inmediatamente después del primer debate (y otros en los debates subsecuentes), uno más después del último escándalo y el último lo más cercano al día $D$ de la elección. Para satisfacer esta demanda, muchos de los estudios deben completar muestras nacionales representativas de todo el electorado, lo cual implica entrevistas

\footnotetext{
${ }^{9}$ Me refiero a procesos y recopilación de información más sofisticada, con ubicación GPS, que se logra al usar tecnología y software con CAPI.
} 
personales, en periodos de investigación muy cortos, que suelen fluctuar entre 4 a 7 (muchas veces para todo el proceso de investigación). Por varias razones -poca inversión en diseño o capacitación, exceso y facilidad de sustituciones de UPM, poca oportunidad de revisitar a individuos seleccionados en muestra, poco tiempo para supervisar y validar el trabajo de campo y otras-, es posible que muchos de los estudios electorales en México ly en otros países de la región) estén generando datos de pobre calidad, confiablidad y validez. En mi experiencia, y dada la extensa y compleja geografía de un país como México, es delicado completar una muestra de 1000 o 1200 casos, dispersa en 100 o 120 UPM, en tan solo cuatro o cinco días de trabajo de campo. Esto en sintesis constituye el problema de la prisa en los estudios electorales, y su consecuencia mayor es que estos mismos estudios son los usados en la generación de conocimiento sobre el comportamiento electoral. Si esto es cierto, el mayor riesgo es que el edificio de conocimiento sobre el comportamiento electoral que estamos construyendo esté sostenido sobre ladrillos inconcretos. Quizás como industria nos toca convencernos y convencer a patrocinadores de estudios electorales que, como dicen, la prisa es mala consejera.

Como muestran los datos del panel 2018, la incidencia de la compra de voto en sus varias modalidades es un fenómeno que afecta a un segmento considerable del electorado y que pudiera estar teniendo un impacto relevante en el comportamiento del electorado. Por lo anterior consideramos que es importante que los estudios electorales futuros consideren su medición y que lo hagan sin prisa y procurando la más alta calidad en la recopilación de los datos. Deseamos también que las condiciones de inseguridad disminuyan y que busquemos formas de hacer nuestro trabajo sin poner en peligro a los entrevistadores y sin tener que sacrificar cobertura o calidad. 